\title{
Student Stress Management and Wellness Programs among Colleges of Pharmacy
}

\author{
Sally A. Arif, PharmD ${ }^{1}$; Kelly Moran, PharmD²; Ana Quiñones-Boex, Ph'; Shareen Y. El-Ibiary, PharmD \\ ${ }^{1}$ Midwestern University, College of Pharmacy-Downers Grove \\ ${ }^{2}$ University of Chicago \\ ${ }^{3}$ Midwestern University, College of Pharmacy-Glendale
}

\begin{abstract}
Objective: To describe the programmatic stress-related interventions that colleges of pharmacy are providing for their students. Methods: A paper-based questionnaire was distributed to 80 college teams who attended two consecutive offerings of the American Association of Colleges of Pharmacy institute focused on promoting student well-being. The five-part questionnaire consisted of: 1) tracking and assessment of perceived student stress levels, 2) the types and formats of stress-coping interventions that are offered, 3) the measured impacts of any stress-coping interventions, 4) the level of faculty/staff training and involvement in student stress remediation, and 5) institutional demographics.

Results: Of the 40 college teams responding to the survey there were similar numbers of private (44\%) and public (56\%) institutions. More than half (57.5\%) reported measuring student stress levels. The most common interventions offered were counseling (95\%), academic advising (82\%), physical exercise support (77\%), and relationship building activities (70\%). Topics offered in the curriculum were most often related to handling substance abuse (50\%), time-management (45\%), and finances (40\%). A majority (79.5\%) of schools reported they do not offer formal training on student stress and mental health to faculty and staff and do not formally assess the impact of stress and coping interventions.

Conclusion: Colleges of pharmacy are addressing student stress and well-being, yet variability exists in terms of assessment, interventions, and didactic offerings. Multiple barriers to improvement remain and mediating barriers and determining assessments for coping and interventions may be next steps for Colleges of Pharmacy.
\end{abstract}

Keywords: student wellness; interventions; stress

\section{INTRODUCTION}

In order for colleges of pharmacy to fulfill their mission of providing high-quality pharmacy education, mental health concerns of pharmacy students should be addressed. In 2011, the Accreditation Council for Pharmacy Education (ACPE) required evaluation of perceived stress by all accredited colleges of pharmacy, stating, "The college or school must have mechanisms to assess and correct underlying causes of ineffective learning experiences. Ideally, colleges of pharmacy assessment processes should include measurement of perceived stress in faculty, staff, and students, and evaluate the potential for a negative impact on programmatic outcomes and morale." 1

There have been several published studies revealing levels of perceived student stress and coping mechanisms of pharmacy students. $^{2-5}$ Typical sources of stress are academic demands, including assignment deadlines and exams, ${ }^{2}$ adjustment to a new environment or culture, ${ }^{3}$ financial burden, ${ }^{2}$ and personal relationships. $^{3} \quad$ Students' coping mechanisms for these stressors range from healthy to unhealthy; these include exercise, talking to friends and family, sleeping, TV/video

Corresponding author: Sally A. Arif, PharmD

Midwestern University, College of Pharmacy

Downers Grove, IL

Phone: 630-515-6466; Email: Sarif@midwestern.edu games, alcohol use, eating, shopping, medication (either prescription or illicit), and smoking. ${ }^{2,3}$ The majority of the studies were conducted within a single institution during the third didactic year, but a few studies surveyed student stress and coping mechanisms at multiple institutions and/or tracked students longitudinally throughout their first three years of programming. ${ }^{3-5}$ Results from longitudinal studies showed that pharmacy students experience the greatest levels of stress during their second didactic year, with stress levels decreasing during the third didactic and fourth experiential years. ${ }^{4,5}$ In addition to studies revealing student stress levels, there have been studies of student resilience, the effectiveness of facultyto-student/preceptor-to-student interventions and meditation. $^{6-8}$ Notably, a student resilience scale was developed focusing on identifying students who have a greater capacity to overcome academic adversities although, the study did not explore the interventions the institution offered. ${ }^{6}$ Largely, the literature identifies that interventions, such as yoga, meditation, and increasing self-awareness and mindfulness through faculty relationships, correlates with decreased student stress levels. ${ }^{7,8}$ A gap in the literature remains in identifying strategies used nationally. The purpose of this study is to describe the programmatic stress-related interventions that colleges of pharmacy are providing for their students. In addition to obtaining a snapshot of commonly employed programmatic interventions to promote student wellness, it is also important to capture the challenges colleges are facing, in delivering or assessing of interventions. 


\section{METHODS}

Following a literature review, a questionnaire was developed by the primary investigator. The 13 item questionnaire contained 5 parts: 1) methods for tracking and assessing perceived student stress levels, 2 ) the types and formats of stress-coping interventions that are offered (3 questions), 3) the measured impacts of any stress-coping interventions (2 questions), and 4) the level of faculty/staff training and involvement in student stress remediation (3 questions), and 5) institutional demographics (5 questions). The questionnaire ranged in response types by asking various open-ended questions, multiple-answer multiple-choice selections, and demographic questions. The survey instrument was reviewed by a group of pharmacy faculty to establish content validity.

A cover letter invitation that included the objective of the study, as well as the anonymous survey tool were distributed to the College of Pharmacy faculty-teams that attended the American Association of Colleges of Pharmacy (AACP) Fall Institute entitled "Student Affairs: Strategies to Promote a Culture of Well-being among Students and Faculty" on October 15th, 2018 in Herndon, VA or the AACP Spring Institute entitled "Strategies to Promote a Culture of Well-being among Students, Faculty and Staff" on May 20th, 2019 in Herndon, VA. The faculty and staff teams attending this institute were invited to complete the questionnaire based on their best knowledge of student wellness institutional interventions being offered at their respective college/school. One survey was collected from each College team at the completion of the Fall and Spring Institute meetings. After all confidential surveys were collected, they were placed in a manila envelope.

Data were analyzed with the use of IBM Statistical Package of the Social Sciences (Armonk, NY, version 25.0). Descriptive statistics were utilized to report on all survey items. This study received exempt status from the Midwestern University Institutional Review Board.

\section{RESULTS}

The response rate was $50 \%(n=40 / 80)$. There was similar representation from public $(56.4 \%)$ and private $(43.6 \%)$ institution faculty teams (Table 1 ). The majority $(92.5 \%)$ of respondents represented 4-year schools. More than half $(57.5 \%, n=23)$ reported currently measuring student stress levels. The respondents' demographics were similar between both the Fall and Spring institutes, as well as representative of the total population that attended both meetings. The most common student interventions offered were individual counseling, academic advising, physical exercise-based classes, and relationship/mentorship building activities amongst peers and with faculty (Table 2). The most common topics covered in the classroom setting included substance abuse, time management, and money/finances (Table 3 ). The majority of schools (79.5\%) do not provide formal training to their faculty and staff in dealing with student stress and mental health related issues. The most reported reasons for the absence of training include "lack of time" and it being considered a low priority for their colleges. Table 4 highlights that most wellness content is taught within courses or within co-curricular programming. The most common themes found as barriers to offering or improving stress-management programing across Colleges of Pharmacy included: time and resources, lack of expertise with tools and assessments, and low priority. Lastly, a very limited number of schools (7.9\%) currently assess the impact of their student stress and coping interventions.

\section{DISCUSSION}

Given that ACPE requires evaluation of perceived stress by all accredited colleges of pharmacy for faculty, staff, and students, it is interesting to note the results of this study. Slightly over half of the sampled pharmacy colleges are measuring student stress levels. Counseling and academic advising are the most offered student interventions at institutions, although it was unspecified if these were delivered at a college or university level. The extent to which those attending student counselling services experience difficulties or how effective counselling is for them is currently unknown. Classes for exercise and yoga are also most commonly incorporated. Less than half of the schools incorporate mindfulness meditation, although this strategy has been shown to reduce stress and anxiety in the higher education literature. ${ }^{9}$

Only a few colleges of pharmacy are actively assessing the effect of the coping interventions. The most common reason cited was due to lacking a proper measurement tool. Future work in the area of student wellness interventions should include evaluating assessment strategies and development of measurement tools specifically for pharmacy students. Tools like the Perceived Stress Scale 10 (PSS10), a validated psychological instrument, can be used to provide a global measure of stress over the past month. ${ }^{10}$ The Brief COPE scale, which measures coping with stressors in life, has also been recently used in pharmacy specific research. ${ }^{11}$

The study results also demonstrated that a limited number of colleges of pharmacy are currently training faculty in methods to support student wellness. Programs, such as Mental Health First Aid, may be ways to help train faculty. ${ }^{12}$ It is important to note that the impact of training, like Mental Health First Aid, can be influenced by when it is delivered or if it is required for all faculty and staff. ${ }^{13}$ Barriers could include time and cost, however, it is important for faculty and staff to have an understanding of stress and coping strategies for the health of the student in addition to the health of the faculty or staff member working with the students. As there are increases in student stress, depression, and other mental health issues, faculty and staff serve a critical role in helping to mediate interventions and refer students for appropriate care. ${ }^{4}$

The American Association of Colleges of Pharmacy actively encourages colleges of pharmacy to promote overall wellness and techniques to manage stress. ${ }^{14}$ Stress, anxiety, and 
depression are all disruptive to effective learning and an effort should be made by schools of pharmacy to provide support. Given the landscape of clinician burnout, focusing on student pharmacist wellness is critical to the profession of pharmacy, to ensure student retention and success, well-being for students and to aid in preventing clinician burnout after graduation..$^{5,15}$ Working together and sharing ideas, is one way of helping to disseminate possible interventions. Of the interventions provided, about $40 \%$ were offering one lecture within a required course. About one-quarter of colleges were offering an elective course regarding well-being. These results indicate that we have more to do in our pharmacy colleges. While the offerings within these courses varied, students were provided with several topics seen to be important when understanding the root of stress and strategies to mitigate stress (e.g. substance abuse, time management, and money/finances).$^{16}$ For example, mindfulness lectures and workshops allow students to practice how grounding techniques, breath work, and journaling can enhance coping with stressful moments. ${ }^{13,17}$ Arming educators with evidence of the positive impact of mindfulness on learning is also important. Mindfulness has been shown to allow students to make connections between concepts taught in different classes, practice patience and presence in the classroom, and improve cognitive functions, such as long-term memory retention. ${ }^{15}$ Programs, such as the AACP Institute on Promoting Faculty, Student and Staff WellBeing, are one step towards increasing awareness, sharing best practices, and implementing interventions. ${ }^{6}$

Barriers must be removed and priority placed on well-being. ${ }^{7,8}$ Institutions must also take a more active role, which should include faculty, administrators, and staff awareness of wellness strategies. More scholarship is needed on educator development around mental health and the level of stigma around mental health in higher education. The data also lays the foundation to investigate the effectiveness of pharmacyspecific pedagogical strategies used in classroom, models of advising/counseling, mentorship, and impact of physical activity on student stress levels.

This study comes with its limitations. These include that the data may include non-response bias due to the response rate. A major limitation of this study is that the number of colleges of pharmacy that completed the survey represent $27.9 \%$ ( 40 out 141) of institutions across the country. There might have been a self-selection for colleges of pharmacy that attended the AACP Institutes. Their attendance might indicate an underrepresentation of programmatic strategies to address student wellness. The responses may not have captured all interventions being offered. Additionally, the survey tool was not designed to capture if interventions were employed to address different sources of perceived stress, which are known to peak at various times in the educational experience. ${ }^{3,5}$ There is also a lack of universal definition to many of the interventions listed in the survey, which further emphasizes the need for attention to this topic. By better understanding strategies that can used for student wellness, the profession can produce more meaningful actionable data.

\section{CONCLUSION}

Colleges of pharmacy are addressing student stress and wellbeing, yet variability exists in terms of assessment, interventions, and program offerings. Multiple barriers to improvement remain and mediating barriers and determining assessments for coping and interventions may be next steps for Colleges of Pharmacy.

\section{Conflicts of Interest: None \\ Funding/support: None}

\section{REFERENCES}

1. Accreditation Council for Pharmacy Education. Accreditation standards and guidelines for the professional program in pharmacy leading to the doctor of pharmacy degree. Retrieved from https://www.acpeaccredit.org/pdf/Standards2016FINAL.pdf. Published February 2, 2015. Accessed April 27, 2020.

2. Beall J, De Hart R, Riggs RM, Hensley J. Perceived stress, stressors, and coping mechanisms among doctor of pharmacy students. Pharmacy. 2015; 3, 344-354. doi: 10.3390/pharmacy3040344

3. Marshall L, Allison A, Nykamp D, Lanke, S. Perceived stress and quality of life among doctor of pharmacy students. Am J Pharm Educ. 2008; 72(6), Article 137. doi: 10.5688/aj7206137

4. Hirsch J, Do A, Hollenbach K, Manoguerra A, Adler D. (2009). Students' health-related quality of life across the preclinical pharmacy curriculum. Am J Pharm Educ. 2009; 73(8), Article 147. doi: 10.5688/aj7308147

5. Votta RJ, Benau E. Predictors of stress in doctor of pharmacy students: Results from a nationwide survey. Curr Pharm Teach Learn. 2013;5(5): 365-372. doi: 10.1016/j.cptl.2013.06.014

6. Chrisholm-Burns M, Spivey C, Sherwin E, Williams J, Phelps S. Development of an instrument to measure academic resilience among pharmacy students. $A m \mathrm{~J}$ Pharm Ed. 2019; 83(6), Article 6986. doi: 10.5688/ajpe6896

7. Shealy S, Worrall C, Baker J, et al. Assessment of a faculty and preceptor development intervention to foster self-awareness and self-confidence. $\mathrm{Am} \mathrm{J}$ Pharm Educ. 2019; 83(7), Article 6920. doi: 10.5688/ajpe6920

8. Lemay V, Hoolahan J, Buchanan A. Impact of a yoga and meditation intervention on students' stress and anxiety levels. Am J Pharm Educ. 2019; 83(5), Article 7001. doi: 10.5688/ajpe7001 
9. Bamber M, Schneider J. Mindfulness-based meditation to decrease stress and anxiety in college students: A narrative synthesis of the research. Educ Research Review. 2016; 18,1-32. doi:10.1016/j.edurev.2015.12.004

10. Cohen S, Kamarck T, Mermelstein R. A global measure of perceived stress. J Health Social Behavior. 1983; 385-396.

11. Sam AT, Muttusamy B, Yee SM, et al. Investigation of stressors affecting a sample of pharmacy students and the coping strategies employed using modified academic stressors scale and brief cope scale: a prospective study. J Young Pharm. 2016; 8(3):122-127 doi:10.5530/jyp.2016.2.12

12. Mental First Aid Website. https://www.mentalhealthfirstaid.org. Published 2020. Accessed April 27, 2021.

13. Chowdhary A, Zlotnikova V, Lucas C, Lonie JM. How do mental health first aid interventions influence patient help-seeking behaviours? A dilemma for pharmacist mental health first aid responders. Res
Social Adm Pharm. 2019; Jan;15(1):106-108. doi: 10.1016/j.sapharm.2018.02.010.

14. Miller M, Boyer C, Emerson M, et al. Report of the 2017-2018 student affairs standing committee. Am J Pharm Educ. 2018; 82 (7) Article 7159. doi:10.5688/ajpe7159

15. National Academy of Medicine. Taking action against clinician burnout a systems approach to professional well-being. Oct. 2019. Available at: https://nam.edu/event/taking-action-againstclinician-burnout-a-systems-approach-toprofessional-well-being-report-release-event/ Published 2019. Accessed April 27, 2021.

16. Cohen J. Social, emotional, ethical, and academic education: creating a climate for learning, participation in democracy, and well-being. Harvard Educ Review. 2006;76(2):201-237. doi:10.17763/HAER.76.2.J44854X1524644VN

17. Bush M. Mindfulness in higher education. Contemp Budd. 2011;12(1): 1476-7953. doi: 10.1080/14639947.2011.564838 
Table 1. College/School Demographics, $\mathbf{N}=\mathbf{4 0}$

\begin{tabular}{ll}
\hline Institution Type & $\mathbf{n}(\%)$ \\
Public & $22(56.4)$ \\
Private & $17(43.6)$ \\
School Mission Orientation & $\mathbf{n}(\%)$ \\
Research & $10(25.6)$ \\
Teaching & $13(33.3)$ \\
Both & $16(41.0)$ \\
Class Size & \\
Average Class Size & $108.0 \pm 46.4$ \\
Program Type & $\mathbf{n}(\%)$ \\
Direct Entry & $1(2.5)$ \\
Four Year & $37(92.5)$ \\
Accelerated & $1(2.5)$ \\
\hline
\end{tabular}


Table 2. Wellness Interventions Offered Among Colleges of Pharmacy, $\mathrm{N}=\mathbf{4 0}$

Intervention Types Offered

Individual Counseling

Academic Advising

Exercise/Fitness Classes

Yoga Classes

Social and Relationship Building Activities with Students

Faculty to Student Mentorship

Education on Wellness

Peer to Peer Mentorship

Social and Relationship Building Activities with Faculty

Mindfulness/Meditation

Reflection

Biofeedback n (\%)

38 (95.0)

33 (82.5)

31 (77.5)

29 (72.5)

$28(70.0)$

26 (65.0)

$25(62.5)$

$24(60.0)$

$23(57.5)$

$18(45.0)$

$17(42.5)$

$2(5.0)$ 
Table 3. Topics Covered During Student Interventions in a Classroom/Didactic Setting, $\mathbf{N}=\mathbf{4 0}$

\begin{tabular}{ll}
\hline Topic Theme & $\mathbf{n}(\%)$ \\
Substance Abuse & $20(50.0)$ \\
Time Management & $18(45.0)$ \\
Money/Financial Planning & $16(40.0)$ \\
Diet & $14(35.0)$ \\
Mindfulness & $14(35.0)$ \\
Sleep & $11(27.5)$ \\
Exercise/Fitness & $10(25.0)$ \\
No topics are covered & $9(22.5)$ \\
Social Engagement & $4(10.0)$ \\
\hline
\end{tabular}

Table 4. Location of Wellness and Student Stress Topics in the Curriculum Among Colleges, $\mathbf{N}=\mathbf{4 0}$ (select all that apply)

\begin{tabular}{lc}
\hline Curriculum Placement & $\mathbf{n}(\%)$ \\
Within a Course - Individual Lecture & $17(42.5)$ \\
Co-Curricular Programming & $15(37.5)$ \\
As an Elective - Live Course & $11(27.5)$ \\
Within an Elective - Individual Lecture & $3(7.5)$ \\
Required - Live Course & $2(5.0)$ \\
Within a Workshop & $2(5.0)$ \\
Required - Online Course & $1(2.5)$ \\
\hline
\end{tabular}

